\title{
An unique case of primary pulmonary cryptococcosis with extensive chest wall invasion
}

A n abnormality was found on the chest radiograph of a 56 year old man during a medical check up. Three months later the bloody sputum and left chest pain were aggravated; the left chest was swollen and pain increased and the patient was admitted a month later. At admission the chest radiograph revealed a left upper lobe mass and a chest CT scan showed extension into the chest wall (fig $1 \mathrm{~A}$ and B). There was tenderness in the left anterior thoracic region and an elastic hard mass with an unclear boundary and a diameter of $5 \mathrm{~cm}$ was palpated but no neurological abnormality was noted. A transbronchial lung biopy specimen was taken but no malignancy was found. Although he had no contact with birds, did not habitually drink alcohol and was not immunosuppressed, Cryptococcus neoformans was detected in lung biopsy samples. Treatment with $200 \mathrm{mg}$ fulconazole was initiated but the symptoms did not improve. A malignant tumour was strongly suspected and the tumour was biopsied by small thoracotomy. After biopsy, drainage was performed, yielding about $50 \mathrm{ml}$ of a reddish brown jelly-like substance. Cryptococcus neoformans alone was found, without any indication of malignancy in the biopsy and drainage samples (fig 2A and B). The symptoms and laboratory findings rapidly improved after drainage and the shadow of the mass disappeared on the chest radiograph and CT scan.

Previous studies have suggested that the most common manifestation of pulmonary cryptococcosis on the chest CT

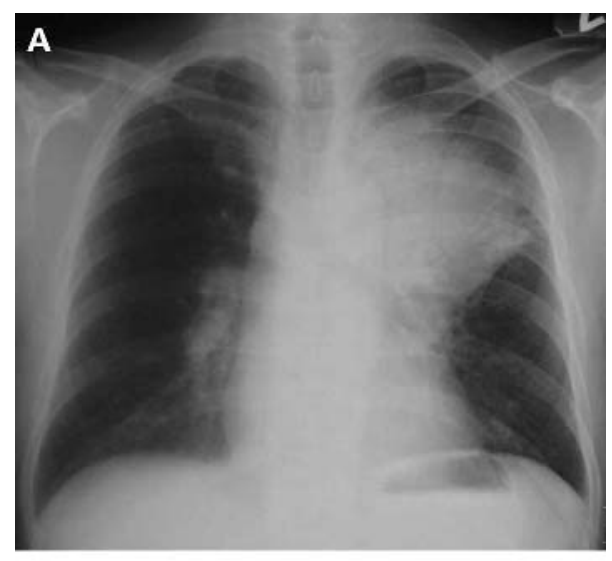

\section{Learning points}

- There is a wide differential diagnosis for mass lesions with extensive chest wall invasion on the chest CT scan, which includes pulmonary cryptococcosis.

- Physicians will probably encounter patterns of pulmonary cryptococcosis on chest CT scans only rarely.
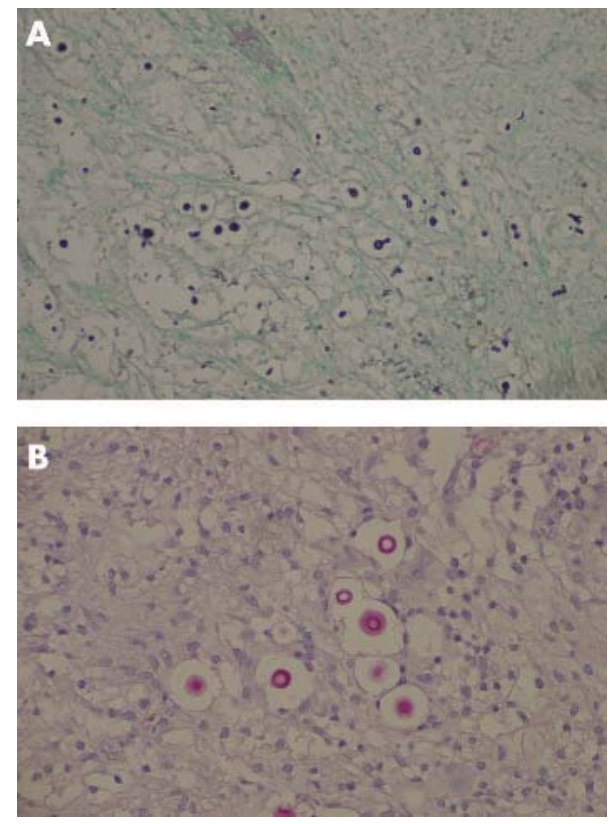

Figure 2 Histological sections of the fumour: (A) Grocott staining; (B) Mucicurrmine staining.

scan is pulmonary nodules, typically $5-20 \mathrm{~mm}$ in diameter. ${ }^{12}$ This patient is an unique case of pulmonary cryptococcosis with extensive chest wall invasion.

\section{ACKNOWLEDGEMENTS}

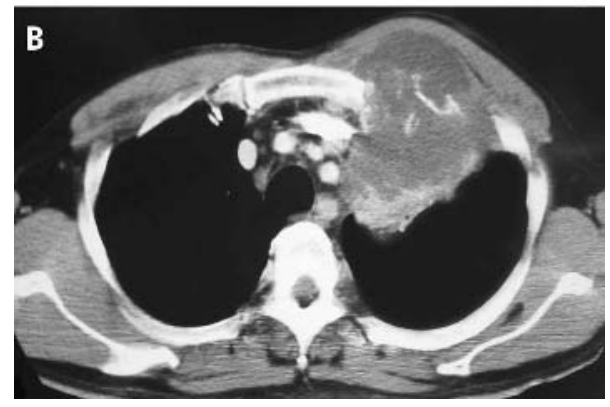

The authors thank Drs N Yoshimura, K Asai, S Kyoh, S Kudoh, K Hirata, and J Yoshikawa for their critical review of the manuscript.

S Mitsuoka, H Kanazawa

Department of Respiratory Medicine, Graduate School of Medicine, Osaka City University, Osaka, Japan

Correspondence to: Dr H Kanazawa, Department of Respiratory Medicine, Graduate School of Medicine, Osaka City University, 1-4-3, Asahi-machi, Abenoku, Osaka 545-8585, Japan; kanazawa-h@med.osaka-cu.ac.jp

\section{REFERENCES}

Figure 1 (A) Chest radiograph on admission showing a left upper lobe mass and (B) chest $C T$ scan on admission showing extension into the chest wall with associated rib destruction.
列 pathologic findings. J Comput Assist Tomogr 2002;26:330-4
2 Murayama S, Sakai S, Soeda $\mathrm{H}$, et al. Pulmonary cryptococcosis in immunocompetent patients: HRCT characteristics. Clin lmaging 2004;28:191-5.

